Peter Rieker, Ellen Höhne und Rebecca Mörgen

\title{
Unterbringung und Betreuung unbegleiteter minderjähriger Geflüchteter in der Schweiz aus Sicht von Fachpersonen
}

Zusammenfassung: Die Unterbringung und Betreuung unbegleiteter minderjähriger Geflüchteter stellt die zuständigen Institutionen und Fachkräfte in der Schweiz vor grosse Herausforderungen, u. a. weil es an einer systematischen Sammlung und Auswertung von Erfahrungen und Wissen zur Arbeit mit dieser Zielgruppe fehlt. Im vorliegenden Beitrag werden auf der Grundlage einer Befragung von Expert*innen die Erfahrungen und Einschätzungen von Fachpersonen, die mit dieser Zielgruppe arbeiten, vorgestellt. Darüber hinaus wird untersucht, inwieweit diese Fachpersonen sich auf die Ergebnisse wissenschaftlicher Studien beziehen.

Schlüsselwörter: UMA (unbegleitete minderjährige Asylsuchende), Unterbringung, Betreuung, Fachpersonen

\section{Placement and Care of Unaccompanied Minor Refugees in Switzerland from a Professional Perspective}

Summary: Lacement and care of unaccompanied minor refugees represent great challenges for the institutions and professionals in charge in Switzerland since a systematic collection and evaluation of experiences and knowledge regarding work with this target group, among other things, is missing. In the contribution at hand, the experiences and assessments of professionals that are working with this target group are presented on the basis of interviews with experts. In addition, it is examined to what extent these professionals refer to results of scientific studies.

Keywords: UMR (unaccompanied minor refugees), placement, care, professionals 


\section{Einleitung}

Im Jahr 2015 ist die Anzahl der unbegleiteten minderjährigen Geflüchteten (im Folgenden: mineurs non accompagné.e.s: MNA), die in der Schweiz Asyl beantragt haben, auf 2736 gestiegen und in den Folgejahren dann wieder abgesunken: 2016 wurden 1997 MNA neu registriert, 2017 noch 733, 2018 401 und 2019 waren es 441 (SEM 2020). Obwohl die Zahl der neuankommenden MNA in den letzten Jahren wieder gesunken ist, gewinnen Fragen ihrer Unterbringung und Betreuung an Bedeutung. Der Zuzug dieser Kinder und Jugendlichen sowie die Fluktuation der Zahl der Einreisenden haben die Institutionen, die für ihre Unterbringung und Betreuung zuständig sind, vor grosse Herausforderungen gestellt. Gleichzeitig wird festgestellt, dass es für die Schweiz an empirisch abgesicherten Erkenntnissen zu den gegenwärtigen Lebensbedingungen von MNA mangelt (Jurt/ Roulin 2016; Keller et al. 2017). Studien zur Situation von MNA, die deren Lebenssituation beleuchten, sind eher für andere Länder wie Schweden (Söderqvist et al. 2014), England (Kohli 2006) oder Deutschland (Thomas et al. 2018; Hargasser 2014; Klingelhöfer / Rieker 2004) zu finden.

Ausgehend von dieser Feststellung fokussiert der Beitrag auf das bei Expert*innen aus verschiedenen Bereichen der Sozialen Arbeit in der Schweiz verfügbare Wissen zu Fragen der Unterbringung und Betreuung von MNA. Hierbei steht die Frage im Fokus, wie diese Fachpersonen die aktuelle Situation der Unterbringung und Betreuung vor dem Hintergrund ihrer Erfahrungen einschätzen. Auf diese Weise soll geklärt werden, inwieweit man in der Fachpraxis in der Schweiz hinsichtlich der Versorgung von MNA an die nationale und internationale wissenschaftliche Fachdiskussion anschliesst und inwiefern die Expert*innen die mitunter schon älteren Befunde dieser Diskussion durch neue Perspektiven ergänzen. Diese Fragen zu klären, ist insofern interessant, als immer wieder deutlich wird, dass man sich in der wissenschaftlichen Diskussion und in der Fachpraxis auf unterschiedliche Wissensbestände bezieht und dass nicht immer ein Austausch zwischen diesen Wissenssystemen gewährleistet ist. Dafür wird sich auf eine Befragung von Expert*innen der Fachpraxis in der Schweiz bezogen, die im Herbst 2018 durchgeführt wurde. Im Folgenden werden zunächst zentrale Aspekte der wissenschaftlichen Fachdiskussion zur Unterbringung und Betreuung von MNA referiert und der Forschungskontext der vorliegenden Überlegungen vorgestellt. Anschliessend werden die von Expert*innen in der Schweiz berichteten Erfahrungen und Einschätzungen vorgestellt und vor dem Hintergrund der veröffentlichten Fachdiskussion diskutiert. 


\section{Wissenschaftliche Fachdiskussion zur Unterbringung und Betreuung unbegleiteter minderjähriger Geflüchteter}

Die institutionelle Unterbringung und Betreuung von unbegleiteten minderjährigen Geflüchteten wird durch unterschiedliche Kontexte der Unterbringung und verschiedene Betreuungskonzepte entscheidend geprägt, daneben spielen aber auch asylpolitische wie asylrechtliche Aspekte eine relevante Rolle. Im Folgenden wird zunächst auf asylpolitische und -rechtliche Aspekte eingegangen, bevor mit Bezug auf den aktuellen Forschungsstand skizzierte Herausforderungen in Bezug auf die Unterbringung und Betreuung von MNAs fokussiert werden.

Die Lebensbedingungen von MNA in der Schweiz werden ganz wesentlich durch die Asylgesetzgebung bestimmt (Mey/Keller 2016). Denn asylrechtlich befinden sich die Kinder und Jugendlichen nicht nur in einem lediglich temporär gesicherten oder ungesicherten Aufenthaltsstatus, was in Hinblick auf das subjektive Wohlbefinden und die Zukunftsplanung mit erheblichen Verunsicherungen verbunden ist, sondern über die Asyl- wie auch Sozialgesetzgebung (Art. 1a BsT d AsylV 1; Art. 14 ZGB) wird die institutionelle Unterbringung und Betreuung durch den Staat übernommen, wenn MNA das 18. Lebensjahr noch nicht vollendet haben (vgl. hierfür auch: Mey et al. 2019, S. $16 \mathrm{ff}$.). Um darüber hinaus der besonderen rechtlichen Stellung von Kindern und Jugendlichen gerecht zu werden, ist im Asylgesetz die besondere Bestimmung der prioritären Behandlung der Asylgesuche von MNA (Art. 17 Abs. $2^{\text {bis }}$ AsylG) wie auch die verpflichtende Ernennung einer Vertrauensperson (Art. 17 Abs. 3 AsylG) während des Asylverfahrens formuliert worden. Nach Zuweisung der MNA in den zuständigen Kanton ist die Ernennung einer zivilgesetzlichen Vertretung vorgesehen. Das Verhältnis zwischen zivilgesetzlichen Vertretungen und asylrechtlichen Vertrauenspersonen junger Geflüchteter ist jedoch weitgehend ungeklärt und es fehlt generell an einer Koordination zwischen den verschiedenen zuständigen Institutionen der Kinder- und Jugendhilfe, des Asylbereichs sowie des Bildungssektors (Mey/Keller 2016, S. 21). Da bei der Zuweisung eines Beistands nach Zivilgesetzbuch resp. einer Vertrauensperson nach Asylrecht vor allem rechtliche Erfordernisse zu beachten sind, verfügen die betroffenen Kinder und Jugendlichen auch hier nur über geringe Teilhabemöglichkeiten (Mey et al. 2019). Auch für junge Geflüchtete, die in anderen europäischen Ländern aufgenommen wurden, wird hervorgehoben, dass es sich in Bezug auf die asylrechtlichen Entscheide, den Zugang zum Bildungssystem und den Aufbau von langfristigen Beziehungen um eine «Kindheit im Wartezustand» (Lewek / Naber 2017) bzw. ein 
«Leben im Limbo» (Nimführ et al. 2017) handele, was sich nachteilig auf die Entwicklung der MNA auswirken könne (Hargasser 2014). Dem entsprechend werden die geringen Möglichkeiten der Teilhabe an für sie relevanten Entscheidungsprozessen als ein zentrales Kennzeichen der Vulnerabilität von MNA hervorgehoben (Andresen / Gerarts 2016; Hargasser 2014; Hochuli 2013), die auch dadurch begünstigt wird, dass sich minderjährige Geflüchtete in einem Spannungsfeld zwischen «einer abweisenden und ausschliessenden Flüchtlingspolitik [...] und der Kinderrechtspolitik» (Jurt / Roulin 2016, S. 100) befinden (Ursprung / Koch 2018). Dieses Spannungsverhältnis bestimme den Einrichtungsalltag, wobei fehlendes asylrechtliches Wissen auf Seiten der Betreuenden einen angemessenen Umgang damit weiter erschwere (vgl. Thomas et al. 2018, S. 219). Zudem können ambivalente rechtsstaatliche Handlungsanforderungen zwischen einer Orientierung an der «Rückkehrfähigkeit» (SODK 2016, S. 21) ins Herkunftsland oder an der sozialen Integration ins Aufnahmeland sowohl bei den betroffenen Kindern und Jugendlichen als auch den Fachkräften zu Unklarheiten in Bezug auf die Ausgestaltung sozialer Beziehungen, der Integration in Ausbildungsangebote als auch der Zukunftsplanung führen, sodass diese häufig nicht befriedigend realisiert werden können (Jurt / Roulin 2016, S. 101f.). Diese Unklarheiten tragen auch dazu bei, dass junge Geflüchtete wichtige soziale Bezüge, z.B. zu Familienangehörigen in anderen Ländern, nicht immer wie gewünscht pflegen können.

Wenn Befunde aus verschiedenen Ländern berücksichtigt werden, ist es sinnvoll, zwischen deren unterschiedlichen Konzeptionen wohlfahrtsstaatlicher Fürsorge für junge Geflüchtete zu differenzieren - auch wenn bislang unklar ist, inwieweit diese sich auf Praxis der Unterbringung auswirken. MNA werden in der Schweiz primär als Flüchtlinge aufgenommen, während sich ihre asylrechtliche Behandlung während der Aufnahmephase in anderen Ländern (z. B. in Deutschland, England, Schweden) stärker am Kindesschutz orientiert (Sandermann et al. 2017, S. 8). Die längerfristige Unterbringung von MNA gilt in der Schweiz (ähnlich wie z.B. in Deutschland) als «Spezialfall», der sich sowohl von der gewöhnlichen Flüchtlingshilfe als auch vom gewöhnlichen Kindesschutz unterscheidet. Demgegenüber wird die Unterbringung in anderen Ländern (z. B. England, Frankreich, Schweden) als Beleg für eine vor allem am Kindesschutz orientierte Behandlung betrachtet (ebd., S. 13). Vor dem Hintergrund der referierten Befunde zur administrativen Behandlung junger Geflüchteter in der Schweiz entsteht - auch im internationalen Vergleich - der Eindruck, dass asylrechtliche Perspektiven zentralen Stellenwert haben und MNA 
hier in erster Linie "Asylsuchende» sind, deren kinder- und jugendspezifischen Bedürfnisse erst an zweiter Stelle Beachtung finden.

Neben den asylpolitischen und -rechtlichen Rahmenbedingungen, die die Lebensbedingungen der Jugendlichen wesentlich mitbestimmen, zeigen sich spezifische Herausforderungen in Hinblick auf die verschiedenen Unterbringungs- und Betreuungskontexte. Forschungsarbeiten, die sich mit den institutionell geprägten Lebenslagen von MNA beschäftigen, machen deutlich, dass sowohl die Unterbringungskonzepte als auch die Betreuungskontexte zu differenzieren sind: MNA werden entweder in Pflegefamilien, in Einrichtungen der Kinder- und Jugendhilfe oder in Asylunterkünften für Erwachsene untergebracht (Brauen / Kantowsky 1982; Klingelhöfer / Rieker 2004; Detemple 2015). Für die Schweiz ist hierbei zu berücksichtigen, dass unbegleitete Minderjährige in den seit 2018 eingerichteten Bundesasylzentren zu ersten Abklärungen asylrechtlicher Belange untergebracht und eingewiesen werden. Von dort aus werden sie dann auf die jeweiligen Kantone und die verschiedenen Unterbringungskontexte verteilt (Mey et al. 2019). Unterscheiden lassen sich die jeweiligen Unterbringungskonzepte der Einrichtungen der Kinder- und Jugendhilfe dahingehend, ob es sich um spezialisierte Einrichtungen wie MNA-Zentren handelt oder junge Geflüchtete gemeinsam mit einheimischen Jugendlichen in allgemeinen Institutionen der Kinder- und Jugendhilfe untergebracht sind. Zudem wird noch auf die nach Kantonen zu differenzierenden Betreuungskontexte und Unterbringungskonzepte hingewiesen (Leuenberger 2010; Keller et al. 2017). Von den auf die jeweiligen Kantone verteilten MNA wurden 201513 \% in Pflegefamilien untergebracht, die meisten von ihnen im Kanton Bern (Seiterle 2017). Für die Schweiz liegen ansonsten keine präzisen statistischen Angaben zur institutionellen Unterbringung geflohener Kinder und Jugendlicher vor.

In Bezug auf die Unterbringung von Jugendlichen mit Fluchterfahrung in Pflegefamilien verweisen Forschungsergebnisse darauf, dass die betroffenen Kinder und Jugendlichen kaum oder gar keine Möglichkeiten haben, über ihre Unterbringung mitzuentscheiden (Seiterle 2019). In einer älteren Studie zeigte sich, dass sie den Kontakt zu ihrer Herkunftskultur nur noch begrenzt oder gar nicht mehr aufrechterhalten können. Dies hängt ganz wesentlich damit zusammen, dass sie dort keine Gelegenheit haben, ihre Muttersprache zu sprechen und regelmässigen Kontakt zu Angehörigen der Herkunftskultur zu pflegen (Sander 1982, S. 162). Zudem wurde dort deutlich, dass die Pflegekinder sich bedingt durch ihr Äusseres dauerhaft als anders und als fremd erleben, auch wenn sie sich noch so 
sehr um Anpassung bemühen, was zu einem negativen Selbstwertgefühl beitragen kann (ebd.). Darüber hinaus berichten sowohl Pflegeeltern als auch junge Geflüchtete von kulturellen Missverständnissen, die zu wechselseitiger Verärgerung und Frustration, mitunter auch zur Aufkündigung des Pflegeverhältnisses und zu häufigeren Umplatzierungen führen können (Brauen / Kantowsky 1982, S. 12 f.; Dahlen 1991). Vor dem Hintergrund solch problematischer Entwicklungen, die für kulturelle und soziale Entfremdungen verantwortlich gemacht wurden, propagieren Nancy Schulz und Ann Sontz den Einsatz von «ethnischen Pflegefamilien», d.h. die Unterbringung geflohener Kinder und Jugendlicher bei Familien, die einen ähnlichen kulturellen Hintergrund wie sie selbst aufweisen. Im Vergleich $\mathrm{zu}$ jungen Geflüchteten, die bei einheimischen Pflegefamilien untergebracht waren, wiesen die bei solchen Pflegefamilien Untergebrachten eine geringere Neigung zu Depressionen auf und berichteten generell von einem besseren Wohlbefinden (Schulz / Sontz 1985). Erklärt werden diese positiven Tendenzen mit der beschützenden und kulturell vertrauten Umgebung einer ethnischen Pflegefamilie, die den Kindern und Jugendlichen den notwendigen emotionalen Rückhalt biete (ebd., S. 25f.) und einen wichtigen Beitrag zur Identitätsfindung leisten könne (Müller-Schlotmann 2014).

Junge Geflüchtete, die in MNA-Zentren untergebracht sind, berichten vor allem aus der Anfangszeit von Verunsicherungen, wenn die sprachliche Verständigung noch schwierig ist und sie sich mit anderen Jugendlichen auf gemeinsame Standards einigen müssen, z. B. die Küchennutzung oder das Putzen betreffend (Hargasser 2014). In diesem Zusammenhang wird einerseits das Bedürfnis nach ethnischer Zugehörigkeit und Zusammengehörigkeit hervorgehoben, dass den Kindern und Jugendlichen ermöglicht, spezifischen kulturellen Praktiken nachzugehen, wie das Begehen religiöser Feste aber auch das Sprechen der gemeinsamen Herkunftssprache (Hargasser 2014, S. 195; Detemple 2015, S. 67f.). Andererseits weist Brigitte Hargasser darauf hin, dass der Kultur im Aufnahmeland zu wenig Rechnung getragen und der ethnischen Zusammensetzung von Fachkräften und Jugendlichen zu wenig Beachtung geschenkt würde (Hargasser 2014, S. 195). Im Zusammenhang mit Beziehungen zu einheimischen Jugendlichen wird vor allem aus der Anfangszeit von Konflikten und Missverständnissen berichtet (Detemple 2015, S. 69; Hargasser 2014) sowie von lediglich oberflächlichen Kontakten und Ablehnung (Thomas et al. 2018, S. 162 f.).

Vor diesem Hintergrund werden beispielsweise durch Hans Dieter Heun oder durch Helga Jockenhövel-Schiecke Betreuungskonzepte 
favorisiert, die speziell solchen Schwierigkeiten entgegenwirken sollen. Einerseits befürwortete man den Einsatz von Betreuungspersonen aus den jeweiligen Herkunftskulturen, andererseits forderte man für junge Geflüchtete eine monoethnische Unterbringung, d.h. die gemeinsame Unterbringung von Jugendlichen aus denselben Herkunftskontexten (Heun et al. 1992, S. 79 ff.; Jockenhövel-Schiecke 1992; Töbel 1993, S. 50). Entsprechende Untersuchungen haben gezeigt, dass enge Freundschaften teilweise bevorzugt zu Jugendlichen aus dem eigenen Herkunftsland aufgebaut werden (Detemple 2015). Allerdings wird eine multiethnische Unterbringung durch viele Jugendliche explizit befürwortet (Weiss et al. 2001, S. $49 \mathrm{ff}$.), auch weil ihnen die ethnische oder kulturelle Herkunft weniger wichtig ist als gemeinsame Interessen oder ein gemeinsames Schicksal, das sie verbindet (ebd.). Mitunter wird auch berichtet, dass Jugendliche in MNA-Zentren eine Art Ersatzfamilie erleben (Detemple 2015, S. 67). Eine vergleichende Untersuchung hat gezeigt, dass Betreuungspersonen und Jugendliche aus einem ähnlichen Herkunftskontext vor allem in den ersten sechs Monaten des Aufenthaltes als wichtig erlebt werden, während Jugendliche, die schon länger in einer entsprechenden Einrichtung leben, andere Prioritäten setzen und sich weniger auf die ethnisch kulturelle Herkunft der für sie wichtigen Personen beziehen (Rieker / Weiss 1999, S. 544).

Obwohl in den letzten Jahren Untersuchungen zur Situation von MNA durchgeführt wurden, die sowohl Fragen der Unterbringung und Betreuung als auch die Lebenssituation der Kinder und Jugendlichen selbst beleuchten, bleibt auffällig, wie wenig Wissen zu diesen Fragen vorliegt. Unklar bleibt vielfach, wie Betreuungspersonen und MNA in Beziehung zu einander treten, wie sich diese Beziehungen entwickeln und inwiefern es sich dabei um Vertrauensverhältnisse handelt. Zu wenig ist auch darüber bekannt, in welcher Weise die Betreuung durch asyl- und ausländerrechtliche Bestimmungen geprägt oder erschwert wird. Zu klären wäre auch, welche Vorstellungen von Kindheit oder Integration in diesem Bereich relevant sind. Vor diesem Hintergrund soll im Folgenden geklärt werden, wie Expert*innen aus der Fachpraxis in der Schweiz die Unterbringung und Betreuung von MNA einschätzen, auf welche Erfahrungen sie sich beziehen und welche Chancen bzw. Herausforderungen sie skizzieren.

\section{Datengrundlage und methodisches Vorgehen}

Grundlage der folgenden Darstellung sind die Daten der Studie «Unbegleitete minderjährige Geflüchtete in institutioneller Betreuung: Chancen und 
Herausforderungen». ${ }^{1}$ Im Kontext dieser Untersuchung wurden im Herbst 201813 Interviews mit 14 Fachpersonen aus fünf Kantonen geführt, die aufgrund ihrer Tätigkeiten als Expert*innen für unterschiedliche Aspekte der Unterbringung und Betreuung von MNA in der Deutschschweiz angefragt worden waren. Interviews wurden mit Mitarbeitenden von Migrationsund Platzierungsinstitutionen, mit Beiständ*innen und Vertrauenspersonen sowie mit NGO-Mitarbeitenden, die im Bereich Recht, Bildungsarbeit oder Partizipation von MNA zuständig sind, geführt. Die Expert*innen blicken auf eine längere Zeit der Arbeit mit MNA zurück, sodass sie unterschiedliche Phasen der Aufnahme und Betreuung von unbegleiteten Minderjährigen in der Schweiz erlebt haben.

Geführt wurden Experteninterviews (Gläser/Laudel 2010), in denen es darum ging, die Erfahrungen, Einschätzungen und Wissensbestände der Befragten möglichst umfassend zu erheben. Dementsprechend erhielten die Expert*innen breiten Raum, um ihre Arbeit und die für sie in diesem Zusammenhang relevanten Beobachtungen, Entwicklungen, Chancen und Herausforderungen zu beschreiben. Zu elf dieser Interviews konnten digitale Audioaufnahmen angefertigt werden, die anschliessend transkribiert wurden. Zusammen mit den ausführlichen Protokollen zu den Gesprächen, die nicht aufgezeichnet werden konnten, wurden diese in der Projektgruppe gemeinsam ausgewertet. Bei der Auswertung orientierte sich die Projektgruppe zunächst am Verfahren des offenen Kodierens gemäss der Grounded Theory, d.h. die zentralen Einschätzungen, Positionen und Erfahrungen der Befragten zu Fragen der Unterbringung und Betreuung von MNA in der Schweiz wurden identifiziert. Anschliessend wurden die auf diese Weise identifizierten Positionierungen zueinander ins Verhältnis gesetzt, um durch Vergleiche und Kontrastierungen das Spektrum der Äusserungen sichtbar zu machen (Strauss 2007, S. 101 ff.).

\section{Einschätzungen von Expert*innen}

Chancen und Herausforderungen werden von den Expert*innen in verschiedenen Bereichen gesehen, auf die im Folgenden eingegangen wird. Zunächst werden Einschätzungen in Bezug auf die asyl- und zivilrechtlichen Vertretungen skizziert. Daran schliesst die Sicht der Expert*innen hinsichtlich der Platzierung von MNA an. Sodann wird auf Überlegungen zur pädagogischen Betreuung eingegangen, bevor Einschätzungen zur sozialen Integration fokussiert werden und mit den Herausforderungen, die sich mit dem Erreichen der Volljährigkeit der geflüchteten Jugendlichen für die Expert*innen ergeben, abgeschlossen wird. ${ }^{2}$ 
Über die Interviews hinweg dominiert die Einschätzung, dass MNA im Vergleich zu einheimischen Kindern und Jugendlichen schlechter gestellt sind - nicht nur weil sie belastende Erfahrungen gemacht haben und zumeist ohne Familie in der Schweiz leben. Benachteiligungen werden vor allem in Hinblick auf die Betreuungs- und Unterstützungsleistungen gesehen, die ihnen als Geflüchtete zustehen, aber auch in Hinblick auf die Chancen schulischer oder beruflicher Bildung. Zum Teil wird in den Interviews sogar von «systematischer Diskriminierung» (E11/509) gesprochen, da junge Geflüchtete in vielerlei Hinsicht die Angebote der allgemeinen Kinder- und Jugendhilfe nicht nutzen können. In den Interviews findet sich daher verschiedentlich die Forderung, junge Geflüchtete sollten in erster Linie als Kinder und nicht als Asylbewerber*innen gesehen werden, um den Erfordernissen des Kindeswohls und des Kindesschutzes angemessen Rechnung tragen zu können (E5/377).

\section{Vertrauenspersonen und Beiständ*innen}

In Hinblick auf das Verhältnis von asylrechtlichen Vertrauenspersonen und zivilgesetzlichen Beiständ*innen werden verschiedene Einschätzungen und Erfahrungen deutlich. Zunächst verweisen die Expert*innen auf die unterschiedlichen Aufgabenfelder von Vertrauenspersonen und Beiständ*innen: Die Vertrauenspersonen sind für die rechtliche Betreuung im Asylverfahren der Minderjährigen verantwortlich. Sie sollen vor und nach der Anhörung beraten, während der Anhörung zugegen sein und sich im Interesse der Unbegleiteten einsetzen, wenn es beispielsweise um die Beweismittelbeschaffung oder um die Ergreifung von Rechtsmitteln geht. Nach Zivilgesetzbuch werden Beiständ*innen für kindesschutzrechtliche Massnahmen eingesetzt, die als administrative Begleitung beim Leben in der Schweiz und als Ansprechpersonen bei Schwierigkeiten und Herausforderungen fungieren. Allerdings werden die Aufgaben je nach Kanton unterschiedlich ausgestaltet, so bestehen zum Beispiel auch Doppelmandate, wenn eine Person sowohl die Rolle der Rechtsvertreter*in im Asylverfahren als auch der Beiständ*in einnimmt. Im Zusammenhang der rechtlichen Vertretung im Asylverfahren äussern die Expert*innen, dass bei hoher Auslastung der Vertrauensperson die Zeit nicht immer ausreiche, um ein angemessenes Vertrauensverhältnis zu den Kindern und Jugendlichen vor der Anhörung aufzubauen (E8/131).

Darüber hinaus machen die Expert*innen deutlich, dass die Beiständ*innen aufgrund der vielfältigen Aufgaben gefordert sein können, sowohl eine Elternfunktion als auch die Rolle einer administrativen Fach- 
person zu übernehmen. Es wird als Aufgabe der Beiständ*in betont, sich nicht in die institutionelle Betreuung am Wohnort der Jugendlichen einzumischen und ihnen gleichzeitig unterstützend zur Seite zu stehen, um jeweils geeignete Unterbringungsformen zu finden (E8/77). Besonders gefordert seien die Beiständ*innen aber nicht nur durch diese Vereinbarung ganz unterschiedlicher Anforderungen, sondern auch dadurch, dass sie mitunter für sehr viele Jugendliche zuständig sind. Je nach Kanton und abhängig von den jeweiligen Fluchtbewegungen werden Zuständigkeiten für ca. 40 Jugendliche, für 60 bis 160 oder für 350 bis 700 Jugendliche berichtet (E2/15; E8/352; E11/818). Angesichts dieser Herausforderungen wird betont, dass die Beiständ*innen sich weit über das übliche Mass hinaus engagieren, z. B. auch dann, wenn die Jugendlichen volljährig werden und sie eigentlich gar nicht mehr zuständig sind. Beistandschaften werden als «Herkulesaufgabe» (E8/266) beschrieben, die angesichts begrenzter Ressourcen so gut wie möglich erledigt würde, bzw. wie es eine Fachperson ausdrückt: «[W]ir versuchen wirklich alles zu geben» (E8/805). Die Möglichkeit, dass Beiständ*innen diesen Spagat bewältigen bzw. angesichts der besonderen Herausforderungen die notwendigen Leistungen erbringen, wird unterschiedlich eingeschätzt. Von einem Teil der Befragten wird hervorgehoben, dass Beiständ*innen für MNA zu wirklichen Vertrauenspersonen werden, sodass diese sich bei Problemen an die Fachpersonen wenden (E9/107). Damit wird der Begriff der «Vertrauensperson» hier nicht im asylrechtlichen Sinne verwendet, sondern in seiner Bedeutung für eine soziale Beziehungsebene. Denn indem die Beiständ*innen zu Vertrauenspersonen für die Jugendlichen werden, könnten Beziehungen zu den Jugendlichen aufgebaut werden, die dann im Rahmen einer Nachbetreuung genutzt werden, wenn sie 18 geworden sind und die Beiständ*innen formal nicht mehr zuständig sind. Für andere Fachpersonen sind Vertrauensaufbau und Unterstützung angesichts der diversen Anforderungen nicht gewährleistet. So berichtet etwa ein Beistand, für die Jugendlichen keine Vertrauensperson sein zu können, da man sich erst bei Problemen kennen lerne und persönliche Kontakte auf Situationen beschränkt seien, in denen Schwierigkeiten bewältigt werden müssten, z. B. ein negativer Asylbescheid (E2/137). Tendenziell werden die Möglichkeiten und Chancen von Beiständ*innen, vertrauensvolle Beziehungen zu den Jugendlichen zu etablieren, eher dann gesehen, wenn diese für eine geringere Anzahl von Jugendlichen zuständig sind. 
Platzierung und Unterbringung von unbegleiteten minderjährigen Geflüchteten in der Schweiz

Hinsichtlich der Frage zu den Platzierungsprozessen von MNA in den verschiedenen Unterbringungs- und Betreuungskontexten in der Schweiz skizzieren die Expert*innen verschiedene Herausforderungen. Hinsichtlich der staatlichen Zuweisungspraxis der MNA an die Kantone, die über das Staatssekretariat für Migration (SEM) erfolgt, wird festgestellt, dass erst seit 2014 spezialisierte Unterkünfte für MNA eingerichtet wurden. Mit Bezug auf den Auf- und Ausbau von kindgerechten und damit geeigneten Unterbringungen für MNA wird einerseits eine fehlende kantonale Harmonisierung hinsichtlich der Betreuungskonzepte kritisiert (E5/402). Andererseits wird darauf hingewiesen, dass die Unterbringung für MNA in einigen Kantonen nicht der zivilgesetzlichen Heimaufsicht unterstellt sei, sondern als Teilbereich des Asylwesens niedrigere Standards als die Einrichtungen der Kinder- und Jugendhilfe aufweist (E11/501). Dementsprechend sei das Kindeswohl und -wohlbefinden häufig nicht gewährleistet (E8/204). Einhergehend mit der Diversität der Unterbringungskonzepte in den 26 Schweizer Kantonen, wie auch den unterschiedlichen kantonalen strukturellen Rahmenbedingungen, wird von einer Expertin hervorgehoben, dass die «Zukunftsperspektiven der Jugendlichen davon abhängen welchem Kanton sie zugewiesen werden» (E11/438). Als Elemente erfolgreicher Fremdunterbringung von MNA nennen die Expert*innen vorhandene Tagesstrukturen, den Zugang zu Schule und anderen Bildungseinrichtungen sowie wenige Wohnortswechsel (E6/21; E8/898).

In Hinblick auf die Platzierung und Unterbringung von MNA kritisieren die befragten Fachleute eine zu geringe Berücksichtigung der besonderen Situation sowie der Bedürfnisse der betroffenen Kinder und Jugendlichen. Gemäss den in den Interviews mehrheitlich vertretenen Positionen, orientieren sich Platzierungsentscheidungen vor allem an betriebswirtschaftlichen Kriterien, denen fachliche Standards der Unterbringung untergeordnet werden (E2/83). So gehe es vor allem darum, die speziell für MNA eingerichteten Institutionen auszulasten. Dies führe teilweise zu hoher erzwungener Fluktuation, was für die Jugendlichen mit mehrfachem Wohnortwechsel innerhalb kurzer Zeit verbunden sein kann (E8/883). Die Belastungen, die dies für die Jugendlichen mit sich bringen und die auch schon zu Suizidversuchen geführt hätten (E8/897), würden von den Verantwortlichen zu wenig beachtet. Welchen Kriterien oder Entscheidungslogiken Platzierungen von einzelnen Kindern und Jugendlichen in bestimmte Einrichtungen folgen, bleibt vielfach intransparent. Deutlich wird durch 
die Expert*inneninterviews jedoch, dass die betroffenen MNA keine Mitsprache bei diesen Entscheidungen haben (E7/814).

In einer ganzen Reihe von Interviews mit den Expert*innen nimmt die Kontrastierung zwischen der Unterbringung in Pflegefamilien und in grossen MNA-Zentren breiten Raum ein. Dabei werden die Pflegefamilien zumeist als besonders vorteilhaft beschrieben. Einerseits wird hervorgehoben, Pflegefamilien hätten ein «riesiges Potential für Integration» (E11/550). Die Befragten beziehen sich hierbei auf gemeinsame Aktivitäten mit der Pflegefamilie sowie auf die vielfältige Unterstützung, die die Jugendlichen im Pflegefamilienalltag erhalten, z.B. bei der Bewältigung schulischer Anforderungen oder bei der Lehrstellensuche. Andererseits wird hervorgehoben, dass die Jugendlichen gerade in Pflegefamilien konstante und verlässliche Bezugspersonen fänden und als Person akzeptiert würden. Demgegenüber wird bemängelt, dass eine individuelle Unterstützung in MNA-Zentren aufgrund des ungünstigen Betreuungsschlüssels nicht möglich sei, sodass sie dort häufig nur «herumhängen» und «verwaltet» würden (E6/658). Sozialpädagog*innen, so sagt ein Experte, seien in MNA-Zentren überfordert und könnten die Jugendlichen nur in Einzelfällen kennenlernen. In einem Interview ist davon die Rede, dass es sich bei einem MNA-Zentrum um einen «Massenschlag» handeln würde, der «bis an den Rand gefüllt» sei (E8/194).

Vor dem Hintergrund dieser Einschätzungen wird von einem Teil der Befragten empfohlen, für MNA die Unterbringung in Pflegefamilien stark auszubauen bzw. diese Unterbringungsform als Standard einzuführen, da sich die Unterbringung in Pflegefamilien bewährt habe (E3/471). Als besonders effektiv wird dabei die Verwandtenpflege eingeschätzt, d.h. die Unterbringung von MNA bei in der Schweiz lebenden Verwandten. Notwendig sei in diesem Zusammenhang allerdings eine intensive fachliche Begleitung - die Rede ist dabei von wöchentlich zwei bis drei ganzen Nachmittagen oder Abenden, an denen mit diesen Pflegefamilien gearbeitet würde (E4/88). Nicht zu empfehlen sei solch eine Unterbringung allerdings dann, wenn die Verwandten traumatisiert seien. Generell wird eine qualifizierte fachliche Begleitung von Pflegefamilien empfohlen, bei denen MNA leben. Dazu gehörten regelmässige Hausbesuche und auch «Intervisionsabende» hätten sich bewährt, d.h. Zusammenkünfte von Pflegeeltern, bei denen Fallbesprechungen stattfinden und ein Erfahrungsaustausch möglich ist (E4/261). Falls traumatisierte Kinder oder Jugendliche bei einer Pflegefamilie untergebracht sind, wird die enge Zusammenarbeit mit psychologischen Fachkräften als unabdingbar angesehen. 
Obwohl die Unterbringung in Pflegefamilien von verschiedenen Expert*innen favorisiert wird, wird auch darauf hingewiesen, dass diese Unterbringungsform in verschiedenen Kantonen nur noch im Ausnahmefall oder gar nicht mehr realisiert wird. Stattdessen würden Kinder und Jugendliche z. B. länger als geplant in Empfangs- und Verfahrenszentren verbleiben und anschliessend vorwiegend bzw. ausschliesslich in MNA-Zentren untergebracht, wo sie lediglich «verwaltet» werden könnten (E6/658). Von 16- bis 18-jährigen Jugendlichen wird berichtet, dass diese bei Kapazitätsengpässen in Unterkünfte für erwachsene Geflüchtete transferiert werden, in denen keine pädagogische Betreuung gewährleistet sei. Selbst Jugendliche, für die - aufgrund von Traumatisierung oder weil sie besonders jung sind - ein besonderer Betreuungsbedarf festgestellt worden sei, würden in grossen Zentren ohne angemessene Betreuung versorgt. Als weiteres Problem wird die ethnische Durchmischung der Kinder und Jugendlichen in den grossen Einrichtungen empfunden. Beispielsweise bei der Zimmerbelegung würden ethnische Kriterien zu wenig beachtet und dies führe mitunter zu Konflikten zwischen Angehörigen verschiedener ethnischer oder nationaler Gruppen (E2/537). Mitunter seien junge Geflüchtete in den MNA-Zentren auch rassistischen Anfeindungen durch andere Jugendliche ausgesetzt (E4/219; E8/554). Allerdings reiche es auch nicht aus, sich an der nationalen Zugehörigkeit zu orientieren. So formuliert in etwa einer der Expert*innen: «[N]ur weil man aus einem Land kommt, heisst das noch lange nicht, dass man miteinander auskommt oder die gleiche Kultur auch im Land hat - das muss man bedenken» (E8/546).

Während ein Teil der Expert*innen die Pflegefamilie als besonders vorteilhafte Unterbringungsform für MNA hervorhebt, thematisieren andere auch Herausforderungen einer Unterbringung in Pflegefamilien. Bemängelt wird eine unzureichende fachliche Begleitung und Unterstützung von Pflegefamilien, die vor allem dann notwendig sei, wenn Pflegeverhältnisse besonders betreuungsintensiv oder durch Belastungen oder Konflikte geprägt seien. Insofern werden Empfehlungen hinsichtlich der Unterbringung in Pflegefamilien dahingehend relativiert, dass sie nicht für alle Kinder und Jugendlichen geeignet seien, vor allem nicht für jene, die einer besonderen fachlichen Betreuung bedürfen, z. B. im Zusammenhang mit Traumatisierungen (E11/555). Allerdings seien in solchen Fällen auch MNA-Zentren oder Jugendheime ungeeignet, sodass die Jugendlichen eigentlich in spezialisierten Einrichtungen untergebracht werden müssten (E8/215). Diese Sonderplatzierungen seien laut eines Experten jedoch 
nicht auf die Gruppe der jungen Geflüchteten ausgerichtet und generell sei es schwierig, eine Kostengutsprache für diese Form der Unterbringung und Betreuung zu erhalten (E8/219).

Auch Erwartungen von Pflegeeltern werden thematisiert, die sich ein «herziges, dankbares Flüchtlingskind aus dem UNICEF-Katalog» wünschten, und dann komme der «belastete, rebellische Teenager», der so wie die Schweizer Jugendlichen leben möchte, was zu Konflikten führen könne (E8/476). Als eine weitere Herausforderung im Pflegefamiliensetting wird betrachtet, dass Pflegeeltern bereits bei der Aufnahme den Abschied von ihrem Pflegekind mitbedenken müssten, da die definitive Dauer des Aufenthalts in der Familie unklar ist (E4/484). Darüber hinaus wird die hohe Belastung betont, denen die Pflegefamilien ausgesetzt seien, die sich rund um die Uhr engagierten und nicht - wie die Fachkräfte in einer Einrichtung - regelmässig Feierabend machen könnten. Schwierig könne es in einem Pflegeverhältnis dann werden, wenn Pflegefamilien ein bestimmtes, z. B. religiös geprägtes Weltbild oder bestimmte Erwartungen an die Jugendlichen hätten und nicht bereit sind, sich mit einer gewissen Offenheit auf die Jugendlichen und ihre speziellen Bedingungen einzulassen (E4/345). Während für die Pflegefamilien also eine hohe Verbindlichkeit beschrieben wird - die als Förderung, aber auch als Einengung erlebt werden könne -, werden grössere Einrichtungen als offene Räume, als Kontexte sozialer Unverbindlichkeit skizziert, was von den Jugendlichen als Vernachlässigung, aber auch als Freiraum erlebt werden könne (E8/488).

\section{Pädagogische Betreuung und Erziehung}

Mängel und besondere Herausforderungen werden von den Expert*innen auch in Hinblick auf die pädagogische Arbeit mit den jungen Geflüchteten festgestellt. In der Betreuung sei man vor allem mit der Bewältigung aktueller Herausforderungen beschäftigt, sodass die längerfristige Umsetzung pädagogischer Konzepte oder Jugendhilfeplanung keinen Platz habe. In Berichten zum konkreten pädagogischen Alltag werden mitunter Konflikte in Hinblick auf spezifische Erziehungsvorstellungen deutlich, mit denen die Kinder und Jugendlichen im Rahmen verschiedener Unterbringungskontexte konfrontiert sind. Diese Konflikte lassen sich z. B. an den Mahlzeiten festmachen, die zu einer bestimmten Zeit gemeinsam eingenommen werden sollen, oder an der Zeit, zu der die Jugendlichen ins Bett gehen sollen. $\mathrm{Zu}$ diesem Thema positionieren sich die Befragten kontrovers. Einerseits vertreten die Expert*innen die Position, dass die Jugendlichen nicht mit solch einseitig festgesetzten strengen Regeln konfrontiert 
werden können, d.h. man könne zu ihnen nicht sagen: «Es wird gegessen was auf den Tisch kommt» (E4/435). Andererseits findet sich aber auch die Einschätzung, solche Regeln würden eine wichtige Orientierung bieten und die Jugendlichen mit den hiesigen Besonderheiten vertraut machen, an welche sie sich auch anpassen müssten (E1/589).

Vereinzelt werden Vorstellungen zu einer pädagogischen Arbeit artikuliert, dieals AlternativenzuVorgabenundSanktionierungen beschrieben werden. In einem Interview ist davon die Rede, dass zunächst ergründet werden müsse, aus welchem familialen und kulturellen Umfeld die Jugendlichen kommen und wie sie die Flucht und die Ankunft in der Schweiz erlebt haben, bevor pädagogisch mit ihnen gearbeitet werden könne (E9/386). Von einer anderen Fachperson wird der Wunsch formuliert, als Pädagogin als Wegbegleiterin für die Kinder und Jugendlichen zu fungieren, wofür man ihre Träume und Ziele kennen müsse. Diese Art der pädagogischen Betreuung sei aufgrund der aktuellen strukturellen Rahmenbedingungen allerdings kaum möglich, da es an den notwendigen finanziellen und personellen Ressourcen fehle (E10/1317). Zudem seien die Möglichkeiten der sprachlichen Verständigung mit den Jugendlichen oft stark eingeschränkt. Einzelne Expert*innen thematisieren den Rückgriff auf Dolmetscher*innen, was jedoch mit grossem logistischen Aufwand verbunden sei (E10/649). Es gäbe Unklarheiten, wer für die Dolmetschertätigkeit finanziell aufkommen soll. In der Folge werde bei Gesprächen zur psychischen Gesundheit von MNA auch auf den Einsatz professioneller Dolmetscher*innen verzichtet, was eine Expertin kritisiert (E11/910).

\section{Integration}

Vor dem Hintergrund der besonderen Situation und der speziellen Herausforderungen, die für die jungen Geflüchteten beschrieben werden, haben Überlegungen zu den Möglichkeiten der Förderung ihrer Integration in den Interviews hohen Stellenwert. Diesbezüglich werden allerdings ganz unterschiedliche Akzente gesetzt. Auf der einen Seite wird hoffnungsvoll auf die «Integrationsagenda» des Bundes verwiesen, die seit Mai 2019 in Kraft ist und den Kantonen mehr finanzielle Ressourcen zur Verfügung stellt. Andererseits werden konkrete Bereiche genannt, die für die Integration der MNA hohe Bedeutung haben. Die berufliche Integration könne über eine berufliche Ausbildung mit Vorlehre erreicht werden (E2/41) und die soziale Integration könne über Mitgliedschaften in öffentlichen Sportoder Kulturvereinen gefördert werden (E4/91). In Hinblick auf die Unterbringung sprechen sich einige Fachpersonen gegen spezialisierte Angebote 
für MNA aus und befürworten integrative Angebote: «Wahre Integration fusst bei mir persönlich nicht auf Separation in dem Sinne, dass ich ein Heim für unbegleitete minderjährige Asylsuchende eröffne, sondern dass ich sie da aufnehme, wo das Leben stattfindet, und das sollte eine gesunde Durchmischung geben»(E10/114). Als Beispiele für solche Angebote, die als integrationsförderlich angesehen werden, werden Pflegefamilien oder Heime genannt, in denen MNA auch in Kontakt zu einheimischen Jugendlichen kommen (E11/694). In einem Interview wird auf eine andere Variante verwiesen, die in der Fachdiskussion als «Binnenintegration» bekannt ist (vgl. Elwert 1982): Indem junge Migrant*innen in Kontakt zu Angehörigen ihrer ethnischen Gruppen gebracht werden, die sich bereits in der Schweiz etablieren konnten, könnten sie wichtige Informationen und Unterstützung in ihrer Muttersprache erhalten, die zunächst Vertrauen und eine persönliche Stabilisierung und dann eine Integration in die verschiedenen Bereiche der Aufnahmegesellschaft ermöglichen (E3/252).

\section{Volljährigkeit}

Mit besonderen Herausforderungen ist für junge Geflüchtete gemäss den befragten Expert*innen das Erreichen der Volljährigkeit verbunden. Denn mit dem Erreichen des 18. Lebensjahres verlieren die Jugendlichen ihre besondere rechtliche Stellung im Asylwesen, d.h. sie werden nicht mehr als «Kinder» prioritär behandelt, sondern so wie andere geflüchtete Erwachsene bzw. Care Leaver. Mit der Volljährigkeit - und z. T. schon früher - erfolgt in der Regel ein Transfer der Jugendlichen von den jeweiligen MNA-Betreuungsstrukturen in die Unterbringungen der Gemeinden. Da es zumeist keine Übergangslösungen gebe, müssten die jungen Geflüchteten dann von einem Tag auf den anderen ihr Leben selbst organisieren und auch administrative Abläufe selbständig gestalten - sei es bei der Krankenkasse, bei der Ausbildungs-, Arbeits- bzw. Wohnungssuche oder bei Verhandlungen mit diversen Ämtern -, ohne dass ihnen dabei pädagogische oder fachliche Unterstützung zustehe. Für die Befragten kommt dies einer Überforderung gleich und der Schritt in die Volljährigkeit sei für junge Geflüchtete daher häufig mit ambivalenten Gefühlen verbunden, bis zu dem Punkt, dass es für sie ein «Angstfaktor» sei, wie es ein Experte formuliert (E7/1028). Für sie stehen unübersehbare Veränderungen an, wenn sie in Kollektivunterkünfte mit erwachsenen Geflüchteten verlegt werden, keinen Kontakt zu den ihnen bisher bekannten Jugendlichen mehr haben und die gewohnten Unterstützungsstrukturen u.a. durch Bezugspersonen wegfallen. Angesichts dieser mit der Volljährigkeit verbundenen Aufgaben, 
Überforderungen und Ungewissheiten wird in einem Interview ein bildlicher Vergleich für die Situation dieser MNA gezogen: «[D]er Teppich wird weggezogen» (E10/540).

Aus Sicht der Befragten bräuchten die Jugendlichen daher zunächst eine angemessene Vorbereitung auf diese Herausforderungen. Zudem wäre es in ihren Augen wichtig, eine individuelle Betreuung dieses Übergangs in die Volljährigkeit zu gewährleisten und ihnen die notwendigen Ressourcen zur Verfügung zu stellen, z.B. einen Computerarbeitsplatz mit Internetanschluss (E3/677). Obwohl Beiständ*innen und pädagogisches Betreuungspersonal formal nicht mehr zuständig sind, engagieren sie sich verschiedentlich auf ehrenamtlicher Basis auch dann noch für die Jugendlichen, wenn diese bereits 18 geworden sind. Auf diese Weise könne teilweise zumindest eine "punktuelle Nachbetreuung» gewährleistet werden, auch wenn die Jugendlichen eigentlich eine intensivere Begleitung bräuchten (E8/440). Auch Pflegefamilien, die sich dann noch weiter um junge Geflüchtete kümmern, erhalten vereinzelt weiter die notwendige Unterstützung durch die zuvor zuständigen Begleitinstitutionen. Allerdings berichten die Expert*innen auch von ersten Einrichtungen, in denen man offizielle Übergangslösungen gefunden habe, sei es, dass die jungen Volljährigen bis zum Lehrabschluss in einem Heim wohnen bleiben können oder das spezielle Angebote für diese Gruppe vorbereitet werden. Allerdings werden diese Lösungen als unzureichend kritisiert (E10/1210). Ein weiteres Problem wird darin gesehen, dass die Kostenübernahme für die Unterstützung der Integration junger Volljähriger vielfach nicht gewährleistet ist (E11/833).

\section{Diskussion}

Die von den Expert*innen der Fachpraxis geäusserten Einschätzungen und Erfahrungen bestätigen in verschiedener Hinsicht die Positionen, die in der wissenschaftlichen Fachdiskussion vertreten werden. Dies trifft ganz allgemein auf die Schlechterstellung von MNA gegenüber einheimischen Kindern und Jugendlichen zu, die mit einer ungenügenden Ressourcenausstattung im MNA-Bereich zusammenhängt und zu einer unzureichenden Betreuung unbegleiteter Geflüchteter beiträgt (Detemple 2015; Hargasser 2014; Jurt/ Roulin 2016). Es werden in den Interviews jedoch auch Positionen vertreten, die den in der wissenschaftlichen Fachdiskussion geäusserten Befunden und Positionen nicht vollumfänglich entsprechen. Überraschend erscheint vor allem die weitgehend positive Einschätzung der Unterbringung in Pflegefamilien, während die Beurteilung von MNA- 
Zentren vorwiegend kritische ausfällt. Die schon seit längerem diskutierten Herausforderungen einer Unterbringung in Pflegefamilien (Brauen / Kantowsky 1982; Dahlen 1991) werden von den befragten Expert*innen ganz offensichtlich geringer gewichtet, als die Vorteile, die bei einer Pflegefamilienunterbringung gesehen werden. Die in der wissenschaftlichen Diskussion weitgehend positiv beurteilte Unterbringung in Familien mit ähnlichen ethnischen Hintergründen (Müller-Schlotmann 2014; Schulz / Sontz 1985) wird nur in einem der Interviews erwähnt, was dafür spricht, dass diese Variante in der Schweizer Fachpraxis keinen grossen Stellenwert hat. Hinzu kommt, dass die Befragten vor allem das «Integrationspotenzial» von Pflegefamilien betonen, während die Befähigung zur Rückkehr ins Herkunftsland nicht erwähnt wird - diesbezüglich hat ganz offensichtlich ein Umdenken stattgefunden.

Während die ethnische Heterogenität, die vor allem mit dem Zusammenleben junger Geflüchteter in MNA-Zentren einhergeht, in der veröffentlichten Fachdiskussion äusserst kontrovers beurteilt wird (Detemple 2015; Rieker / Weiss 1999), wird sie durch die Expert*innen überwiegend als Belastung eingeschätzt. Auf Anregungen und Potenziale, die mit einer multiethnischen Unterbringung verbunden sein können, beziehen sich die Befragten kaum. Diese Diskrepanz könnte Ausdruck unterschiedlicher Prioritäten von Wissenschaft und Fachpraxis sein, d.h. dass man in der Wissenschaft vor dem Hintergrund theoretischer Positionen solch ein Zusammenleben positiver beurteilt als in der Praxis, wo diesbezüglich vor allem konkrete Schwierigkeiten im Vordergrund stehen. Zu der kritischen Sichtweise auf die Unterbringung in MNA-Zentren, die in den Interviews ausgeprägt ist, trägt zudem die Einschätzung bei, dass die Möglichkeit, pädagogische Vertrauensbeziehungen zu unterhalten und angemessene Betreuung zu gewährleisten, durch bürokratische Abläufe und die Orientierung an betriebswirtschaftlichen Kriterien belastet sei. Demgegenüber werden potenzielle Vorzüge dieser Unterbringungsform - z. B. Professionalität des Fachpersonals, Zusammengehörigkeitsgefühl unter den Bewohner*innen (vgl. Hargasser 2014; Weiss et al. 2001) - kaum erwähnt. Ebenfalls nicht thematisiert wird von den befragten Fachpersonen das Spannungsverhältnis zwischen Selbständigkeit und Hilfebedarf, das vor dem Hintergrund der Fragen nach unterschiedlichen Kindheitskonzepten (Liebel 1999) in der wissenschaftlichen Fachdiskussion immer wieder als Herausforderung beschrieben wird (Theilmann 2005; Hargasser 2014; Detemple 2015). Es kann vermutet werden, dass diese Frage, ungeachtet 
ihrer entscheidenden Bedeutung, angesichts drängender handlungspraktischer Herausforderungen in den Hintergrund gerät.

Verschiedene Themen, die in den Interviews grossen Stellenwert haben, haben in der bisherigen wissenschaftlichen Fachdiskussion kaum oder nur teilweise Beachtung gefunden. Genannt wird vor allem der Übergang in die Volljährigkeit, der bei MNA nur in seltenen Fällen unterstützt oder abgefedert wird, sodass in dieser Situation eine Überforderung der jungen Erwachsenen unvermeidbar erscheint. Für die Schweiz sind zwar die ambivalenten Gefühle der jungen Geflüchteten dokumentiert, die als Volljährige die Jugendhilfeangebote oder die Einrichtungen des Asylwesens, die speziell für Jugendliche eingerichtet wurden, verlassen müssen und dann nicht mehr auf die umsorgende Unterstützung zurückgreifen können (Gilliéron / Jurt 2017, S. 143 ff.), allerdings war bisher unklar, welche Relevanz Fachkräfte diesem Aspekt beimessen. Auch die Aufgaben und der Stellenwert von Beiständ*innen und Vertrauenspersonen sowie ihre Beziehungen zu den jungen Geflüchteten wurden bisher eher selten diskutiert. Vor diesem Hintergrund ist es interessant, dass sie von den befragten Expert*innen als potenziell wichtige Unterstützung für MNA skizziert werden, die ihre Funktion aufgrund hoher Fallzahlen, für die sie zuständig sind, häufig nicht befriedigend ausüben könnten. Zudem wird in den Interviews deutlich, dass in der Schweiz inzwischen spezialisierte Angebote entwickelt wurden, um den besonderen Herausforderungen der Unterbringung und Betreuung von MNA gerecht zu werden, z. B. Intervisionsabende für Pflegefamilien, institutionalisierte Übergangslösungen für junge Volljährige, Mentoringprogramme, in denen Migrant*innen als «Brückenbauer» fungieren, um auf diese Weise die Integration zu fördern. In verschiedener Hinsicht scheinen die befragten Expert*innen also über ein Spezialwissen zu verfügen, das in der wissenschaftlichen Fachdiskussion bisher noch nicht angemessen präsent ist.

\section{Literatur}

Andresen, Sabine \& Gerarts, Katharina (2016). Kindheitsforschung und ihre Zugangsmöglichkeiten zu geflüchteten Kindern. In: Jörg Fischer \& Gunther Graßhoff (Hrsg.), Unbegleitete minderjährige Flüchtlinge. «In erster Linie Kinder und Jugendliche!» Sozialmagazin. Special Vol. 1. Weinheim: Beltz Juventa, S. 154-163.
Brauen, Martin \& Kantowsky, Detlef (1982). Einleitung. In: Martin Brauen \& Detlef Kantowsky (Hrsg.), Junge Tibeter in der Schweiz. Studien zum Prozess kultureller Identifikation. Diessenhofen: Rügger, S. 7-33.

Dahlen, Winfried (1991). Probleme beim Umgang mit indochinesischen Kindern und Jugendlichen in deutschen Pflege- 
familien. In: Materialien zur Heimerziehung, 1(2), S. 41-43.

Detemple, Katharina (2015). Zwischen Autonomie und Hilfebedarf. Unbegleitete minderjährige Flüchtlinge in der Jugendhilfe. Baltmannsweiler: Schneider Hohengehren.

Elwert, Georg (1982). Gesellschaftliche Integration durch Binnenintegration? In: Kölner Zeitschrift für Soziologie und Sozialpsychologie, 34, S. 717-731.

Gilliéron, Gwendolyn \& Jurt, Luzia (2017). Ein Übergang mit Herausforderungen: Erfahrungen ehemaliger, unbegleiteter, minderjähriger Asylsuchenden. In: Soziale Passagen, 9(1), S. 135-151.

Gläser, Jochen \& Laudel, Grit (2010). Experteninterviews und qualitative Inhaltsanalyse. Wiesbaden: Springer VS.

Hargasser, Brigitte (2014). Unbegleitete minderjährige Flüchtlinge. Sequentielle Traumatisierungsprozesse und die Aufgabe der Jugendhilfe. Frankfurt am Main: Brandes \& Apsel.

Heun, Hans-Dieter; Kallert, Heide \& Bacherl Clemens (1992). Jugendliche Flüchtlinge in Heimen der Jugendhilfe. Situation und Zukunftsperspektiven. Freiburg: Lambertus.

Hochuli, Marianne (2013). Kinder und Jugendliche in den Zwängen des Asylrechts. Positionierung der Caritas zum Spannungsfeld zwischen Kinderrechten und Schweizer Asylpolitik. Luzern: Caritas Schweiz.

Jockenhövel-Schiecke, Helga (1992). Junge Menschen auf der Flucht. Kindeswohl zwischen Bleibemöglichkeit und Rückkehr. In: Landesjugendamt Rheinland (Hrsg.), Junge Menschen auf der Flucht, S. $14-32$.

Jurt, Luzia \& Roulin, Christophe (2016). Begleitung und Betreuung von unbegleiteten minderjährigen Asylsuchenden: Die Wahrnehmung von Care-Arbeit aus Sicht der Klientinnen und Klienten. In: Diskurs Kindheits- und Jugendforschung, 11(1), S. 99-111.

Keller, Samuel; Mey, Eva \& Gabriel, Thomas (2017). Unaccompanied Minor Asylum-
Seekers in Switzerland. A Critical Appraisal of Procedures, Conditions and Recent Changes. In: Social Work \& Society, 15(1), S. 1-18.

Klingelhöfer, Susanne \& Rieker, Peter (2004). Junge Flüchtlinge in Deutschland. Informationen - Forschungsstand - Forschungsbedarf. In: JugendBeruf-Gesellschaft. Zeitschrift für Jugendsozialarbeit, 55, S. 100-108.

Kohli, Ravi K. S. (2006). The Sound of Silence: Listening to What Unaccompanied Asylum-Seeking Children Say and Do not Say. In: The British Journal of Social Work, 36(5), S. 707-721.

Leuenberger, Peter (2010). Nur wenige haben so viel Glück. In: Fluchtpunkt, 48, S. 2-3.

Lewek, Mirjam \& Naber, Adam (2017). Kindheit im Wartezustand. Studie zur Situation von Kindern und Jugendlichen in Flüchtlingsunterkünften in Deutschland. Köln: Deutsches Komitee für UNICEF. https://www.unicef.de/ informieren/aktuelles/presse/2017/ studie-fluechtlingskinder-indeutschland/137440 [11.12.2017].

Liebel, Manfred (1999). Kinder der Dritten Welt. Handlungspotenziale und Überlebensstrategien. In: WOGE e. V. \& Institut für soziale Arbeit e.V. (Hrsg.), Handbuch der Sozialen Arbeit mit Kinderflüchtlingen. Münster: Votum, S. 36-45.

Mey, Eva \& Keller, Samuel (2016). Im Schnittfeld von Asylpolitik und Kindesschutz. Dringliche Herausforderungen im Umgang mit unbegleiteten minderjährigen Asylsuchenden. In: SozialAktuell, 4, S. 20-22.

Mey, Eva; Keller, Samuel; Adili, Kushtrim; Bombach, Clara, Eser Davolio, Miryam; Gehring, Milina; Kehl, Konstantin \& Müller-Suleymanova, Dilyara (2019). Evaluation des UMA-Pilotprojektes. Befunde zur kindes- und altersgerechten Unterbringung und Betreuung von unbegleiteten minderjährigen Asylsuchenden in den Zentren des Bundes. Zürcher Hochschule für angewandte Wissenschaften. https://www.sem.admin.ch/ dam/data/sem/aktuell/news/2019/2019- 
06-11/bericht-evaluation-uma-d.pdf [17.09.2019].

Müller-Schlotmann, Richard (2014). Pflegefamilien mit Migrationsgeschichteeine Ressource in der Jugendhilfe. In: Forum Erziehungshilfen, 20, S. 78-82.

Nimführ, Sarah; Otto, Laura \& Samateh, Gabriel (2017). Gerettet aber nicht angekommen. Von Geflüchteten in Malta. In: Sabine Hess, Bernd Kasparek, Stefanie Kron, Mathias Rodatz, Maria Schwertl \& Simon Sontowski (Hrsg.), Der lange Sommer der Migration: Grenzregime III. Assoziation A, S. 137-150.

Rieker, Peter \& Weiss, Karin (1999). Monoethnische oder multiethnische Unterbringung? In: WOGE e.V. \& Institut für soziale Arbeit e. V. (Hrsg.), Handbuch der Sozialen Arbeit mit Kinderflüchtlingen. Münster: Votum, S. 539-546.

Sander, Reinhard (1982). Ergebnisse der Befragung. In: Martin Brauen \& Detlef Kantowsky (Hrsg.), Junge Tibeter in der Schweiz. Studien zum Prozess kultureller Identifikation. Diessenhofen: Rüegger, S. 91-192.

Sandermann, Philipp; Husen, Onno \& Zeller, Maren (2017). European Welfare States Constructing "Unaccompanied Minors". A Comparative Analysis of Existing Research on 13 European Countries. In: Social Work \& Society, 15(2), S. 1-18.

Schulz, Nancy \& Sontz, Ann (1985). Voyagers in the Land: eine Studie über unbegleitete südostasiatische Flüchtlingskinder. In: Menschen unterwegs, 2, S. 3-39.

Seiterle, Nicolette (2017). Ergebnisbericht Bestandesaufnahme Pflegekinder Schweiz 2015. Zürich: PACH - Pflege- und Adoptivkinder Schweiz.

Seiterle, Nicolette (2019). Pflegefamilien erleichtern Flüchtlingen die Integration. Zürich: PACH - Pflege-und Adoptivkinder Schweiz. Online verfügbar unter: https://pa-ch.ch/wp-content/ uploads/2019/07/Netz_Pflegefamilien_ MNA.pdf (28.08.2019).

SEM Staatssekretariat für Migration (2019). Unbegleitete minderjährige Asylsuchende in der Schweiz. https://www.sem.admin. ch/dam/data/sem/publiservice/ statistik/asylstatistik/statistiken_uma/ uma-2019-d.pdf [24.01.2020].

Söderqvist, Åsa; Sjöblom, Yvonne \& Bülow, Pia (2014). Home Sweet Home? Professionals' Understanding of "Home" Within Residential Care of Unaccompanied Youths in Sweden. In: Child and Family Social Work, 21(4), S. 591-599.

SODK Konferenz der kantonalen Sozialdirektorinnen und -direktoren (2016). Empfehlungen der Konferenz der kantonalen Sozialdirektorinnen und Sozialdirektoren. Zu unbegleiteten minderjährigen Kindern und Jugendlichen aus dem Asylbereich. http://www.sodk.ch/ aktuell/empfehlungen/ [05.06.2017].

Strauss, Anselm L. (2007). Grundlagen qualitativer Sozialforschung. Datenanalyse und Theoriebildung in der empirischen soziologischen Forschung. München: Fink

Theilmann, Susanne (2005). Lernen, Lehren, Macht. Zu Möglichkeitsräumen in der pädagogischen Arbeit mit unbegleiteten minderjährigen Flüchtlingen. Oldenburg: BIS Verlag. http://oops.uni-oldenburg. de/519/1/theler05.pdf [05.06.2017].

Thomas, Stefan; Sauer, Madeleine \& Zalewski, Ingmar (2018). Unbegleitete minderjährige Geflüchtete. Ihre Lebenssituationen und Perspektiven in Deutschland. Bielefeld: transcript.

Töbel, Ingrid (1993). Längerfristige Unterbringung am Beispiel des multinational belegten Jugendheimes «Haus Margret». In: Helga Jockenhövel-Schiecke (Hrsg.), Unbegleitete Flüchtlingskinder in Grossstädten der Bundesrepublik Deutschland. Frankfurt am Main: Internationaler Sozialdienst, S. 46-52.

Ursprung, Georgiana \& Koch, Patricia (2018). Zwischen Asyl- und Kinderrecht. Entwicklungen in der Betreuung von unbegleiteten Minderjährigen in der Schweiz. In: SozialAktuell, 11, S. 24-27.

Weiss, Karin; Enderlein, Oggi \& Rieker, Peter (2001). Junge Flüchtlinge in multikultureller Gesellschaft. Opladen: Leske+ Budrich. 


\section{Anmerkungen}

1 Das Forschungsprojekt wird seit 2018 am Institut für Erziehungswissenschaft der Universität Zürich durchgeführt. Es wird vom Schweizerischen Nationalfonds gefördert und ist Teil des Nationalen Forschungsprogramms NFP 76. Im Projekt arbeiten Ellen Höhne (wissenschaftliche Mitarbeiterin), Rebecca Mörgen (wissenschaftliche Mitarbeiterin) und Peter Rieker (Projektleitung).
2 Bezüge zu den Expert*inneninterviews werden jeweils in Klammern angeführt, wobei die Nummer des jeweiligen Interviews und die Zeilenangabe im Transkript angegeben sind, z. B. E4/17. Verwiesen wird in der Regel jeweils auf die Interviewpassagen, in denen die genannten Positionen am deutlichsten formuliert sind.

\section{Biografische Angaben}

Peter Rieker ist Professor für Ausserschulische Bildung und Erziehung am Institut für Erziehungswissenschaft der Universität Zürich. Er forscht zu Kindheit und Jugend, zu Abweichendem Verhalten und Extremismus sowie zu politischer Partizipation, Migration und interethnische Beziehungen. Zudem arbeitet er zu Methoden der qualitativen Sozialforschung und zu multimethodischer Forschung. Kontakt: prieker@ife.uzh.ch.

Ellen Höhne ist Doktorandin am Lehrstuhl für Ausserschulische Bildung und Erziehung am Institut für Erziehungswissenschaft der Universität Zürich und forscht zu sozialen Beziehungen unbegleiteter minderjähriger Flüchtlinge im Ankunftskontext Schweiz. Darüber hin- aus interessiert sie sich für Erinnerungsprozesse- und politiken und arbeitet zu Rassismus und Justiz. Kontakt: ellen. hoehne@ife.uzh.ch.

Rebecca Mörgen ist wissenschaftliche Mitarbeiterin (Post-Doc) am Lehrstuhl für Ausserschulische Bildung und Erziehung am Institut für Erziehungswissenschaft der Universität Zürich. Sie forscht zu Kindheit und Jugend unter Bedingungen der Flucht*Migration; sozialer Teilhabemöglichkeiten; zu qualitativen Migrations-, Ungleichheits- und Professionsforschung im Kontext Sozialpädagogik. Zudem arbeitet sie zu Methoden der qualitativen Sozialforschung, vor allem zu Ethnographie. Kontakt: rmoergen@ife.uzh.ch. 